Z. Wahrscheinlichkeitstheorie verw. Gebiete $54,117(1980)$
Zeitschrift für

Wahrscheinlichkeitstheorie und verwand te Gebiete

(C) by Springer-Verlag 1980

\title{
Correction to Finitary Coding of Markov Random Fields
}

Andrés del Junco

Z. Wahrscheinlichkeitstheorie verw. Gebiete 52, 193-202 (1980)

Lemma 2.2 of the article "Finitary Coding of Markov Random Fields" which appeared on pages 193-202 of volume 52 of this journal is false as stated. The difficulty is that "conditioning on $X(\hat{x})$ " involves conditioning on non-occurrences of $M$ as well as occurrences of $M$ and boundary values. This can be circumvented by thickening the set of boundary values. Specifically, if $M$ is an atom of $P^{B(k)}$ and $E_{0}$ is the Rohlin set with disjoint images $\sigma^{i} E_{0}, i \in B\left(N_{0}\right)$, then 0 -skeleta should be defined so as to note values of the Markov random field on $B\left(N_{0}\right)-B\left(N_{0}-K\right)$, a "boundary" of thickness $K$. Thus no occurrence or nonoccurrence of $M$ can influence both the inside and the outside of the boundary at once, so that Lemma 2.2 goes through. Since $N_{0}$ can be chosen large independent of $K$, the rest of the argument goes through without any change. 